\title{
A Novel Network Selection Mechanism for Vehicle-to-Infrastructure Communication
}

\author{
Emmanuel Ndashimye*, Nurul I Sarkar \\ School of Engineering, Computer \\ and Mathematical Sciences \\ Auckland University of Technology \\ Auckland, New Zealand \\ \{endashim, nurul.sarkar\}@aut.ac.nz
}

\author{
Sayan Kumar Ray \\ Faculty of Business and \\ Information Technology \\ Manukau Institute of Technology \\ Auckland, New Zealand \\ Sayan.Ray@manukau.ac.nz
}

\begin{abstract}
The commercial deployment of 5G networks require heterogeneous multi-tier, multiple radio access technologies (RATs) to support vehicle-to-infrastructure (V2I) communication with diversified services. Vehicles may need to cross a number of heterogeneous networks of various sizes before reaching the destination. Due to high-speed travel, vehicles may quickly move in and out of the network coverage areas while performing handover. Fast and efficient selection of an appropriate underlying network is critical for seamless handover performance. In this paper we propose a novel network selection mechanism for improved handover performance in V2I communication over heterogeneous wireless network. The idea is for vehicles to self-evaluate a candidate list of access points (AP) that are located in the vehicle movement direction and select the best underlying candidate network based on key criteria, like, the distance between target candidate and the trajectory of the vehicle movement as well as the vehicle mobility information. Fuzzy logic inference system is used to decide whether a target candidate is suitable for handover. Experimental results show that for a vehicle moving at $30 \mathrm{~km} / \mathrm{h}$, an AP of $100 \mathrm{~m}$ radius should be located at less than $30 \mathrm{~m}$ from the road, while this distance is limited to $15 \mathrm{~m}$ when the vehicle speed is $60 \mathrm{~km} / \mathrm{h}$.
\end{abstract}

Keywords: V2I; Multi-tier network; heterogeneous RATs Macro-cell; Small cells; handover; Fuzzy logic.

\section{INTRODUCTION AND MOTIVATION}

While Vehicle-to-Vehicle (V2V) communication is supported by IEEE $802.11 \mathrm{p}$, also known as the WAVE (Wireless Access in Vehicular Environments) protocol, V2I communications is also expected to be supported by the different radio access technologies (RATs) [1]. According to a recent report from Gartner Inc, by 2020, worldwide there will be around 250 million vehicles on the roads with wireless network connections in them [2]. Presumably, most of these vehicles will maintain connections with the roadside infrastructure and would perform frequent vertical handovers (switching to access point of different technology) on the move from one underlying network to another. To maintain the acceptable quality of services (QoS) for on-going communication, vertical handovers need to be fast, seamless and reliable. Generally speaking, vertical handovers are affected by handover delays, packet losses (or even call disruptions) and frequent ping pong effects [3].
The future $5 \mathrm{G}$ networks will experience a seamless coexistence of underlying cells of multiple sizes (macro, pico, femto) and diverse access technologies (UMTS, HSPA, WiMAX, LTE, and WiFi). Vehicles moving through such diversified networks, will have to overcome challenges such as, frequent unwanted handovers, selection of inappropriate candidate networks for handovers, delay in completing the lengthy handover procedure leading to failure in handovers. All these will hamper the overall handover performance affecting the seamless mobility of vehicles [4].

In multi-tier network environments (with underlying networks of multiple sizes), vehicles need to perform seamless handovers between the diverse underlying networks of different sizes and one of the key challenges is to design efficient downward handover mechanisms, when switching from large (e.g., macro) to small cell networks (e.g., pico / femto). Presumably, the macro-cell network is assumed to be available anywhere, anytime and thus, a vehicle connected to the macro cell, can perform handover to a small cell without, apparently, running at a risk of losing the existing connectivity before the completion of the handover [5]. The primary motivation for such downward handovers is associated with the usage cost and increased data rate offered by small cells, as compared to large-scale networks such as cellular networks. However, two critical issues are associated with such handovers. Firstly, the visiting time that the vehicle stays in a small cell should be long enough to complete all required handover procedures including exchange of the signaling messages and resuming IP connectivity with the new cell (network) and secondly, after handover, the dwelling time of the vehicle in the small cell should be reasonable enough at least make use of the high data rate offered by small cell network for effective communication.

To facilitate fast and seamless selection of available networks the Third Generation Partnership Project (3GPP) Release 8 has introduced the Access Network Discovery and Selection Function (ANDSF) existing within the Evolved Packet Core (EPC) [6]. ANDSF helps an user equipment (UE) to automatically discover and select the most suitable underlying access network based on certain priorities and policies predetermined by the network operators.

This paper proposes a fully vehicle-controlled and 
network-assisted selection mechanism suitable for handover in a heterogeneous wireless network environment, which includes LTE macro cells and WLAN enabled RSUs. The main objective is to minimize the overall handover delay by performing network selection in advance, and to avoid unsuccessful handovers resulting in Ping-Pong effects. Our proposed network selection mechanism, relies on the information received form the ANDSF server, particularly, the geographical locations of the access points (AP). Based on this information, the vehicle first determines the candidate APs located in its movement direction and then adds the suitable candidate APs in its candidate list. The probability of adding an AP/SCBS to the candidate list is directly associated with the duration the vehicle may dwell under the coverage area of that particular candidate. The vehicle selfcalculates this dwelling time from its speed of movement and the coverage area of the candidate network. Finally, the vehicle selects the most promising candidate AP for which its dwelling time is the maximum. In this context, two terminologies frequently used in the paper need mentioning: (a) candidate networks imply underlying networks that have potential to be selected as the target network for handover and (b) target network/target candidate network implies the most suitable/appropriate candidate network selected for the handover activity. The remainder of this paper is organized as follows. In section II, we discuss related works, while in Section III the proposed network selection mechanism is described. In section IV simulation results are discussed to evaluate the performance of the proposed mechanism and finally, Section V concludes the paper.

\section{RELATED WORK}

A considerable number of research activities have been carried out in context of network selection and notable solutions have been proposed in the relevant literature. Generally, the unique characteristic of vehicular network is high mobility. The recent search presented in [5,7-9] have focused on devising mobility aware network selection techniques, where the direction of movement and node residence time are the most influential decision factors. These two factors are generally adopted with combination of resource availability and usage cost to efficiently select proper candidate network for handover. A location aware network selection technique is proposed in [5], which selects the proper target network based on the estimated residence time under the service area of the selected network. Similarly, the handover scheme in Mobile WiMAX is proposed in [7], where the geographical position of neighboring base station and the direction of mobile terminal are used to determine the short list of potential candidate networks, from which the best candidate is selected based on two criteria, namely, orientation matching and received signal strength. The main weakness with both techniques is that the resource availability is overlooked, which may result in increased handover drop probability. To overcome this weakness, the study in [8], enhanced the mechanism proposed in [7] by introducing the current load of the target BS as one of decision factors to improve the handover stability. In the same way, the network selection approach in [9], proposed an access index function, where important influential factors are: data rate, direction of movement and the vehicle residence time. Furthermore, the effective target network is selected based on network usage fee as well as security factor.

on the other hand, a number of studies have applied artificial intelligent based network selection approaches such as fuzzy logic systems. In [10], authors proposed a fuzzy Q-learning based vertical handover for vehicular network, which decides the optimal network based on four input parameters: RSS value, Vehicle speed, data quantity and the number of users associated with the target network. The proposed algorithm integrates WLAN (WAVE protocol) with cellular networks; where the objective is to ensure that WLAN supply long service time than cellular network. The suggested mechanism has a real time learning capability. However it is only designed for infotainment applications it is not suitable for delay sensitive applications. A similar scheme was also proposed in [11], but the network usage cost and transmission time were the main variables in fuzzy set, whose membership degree is influencing vertical handover. The simulation results show that the proposed algorithm improves V2I vertical handover in terms of transmission time and cost, compared to conventional RSS-based handover.

The common drawback of fuzzy logic based solutions is linked with increased fuzzy if-then rules, which may introduce processing delay. However, for few inputs, fuzzy logic could be an efficient tool due to its ability to deal with non-linearity and uncertainties systems [12]. In the present paper, the fuzzy logic system is utilized to implement the proposed approach since we have only two inputs whose values are not precise.

\section{Proposed Network Selection Mechanism}

Generally, a handover procedure has three phases: network discovery, network selection and handover execution [13]. In heterogeneous wireless networks, selection of the candidate network for handover is a complicated and time consuming process. Whenever, a vehicle on the move from one location to another, decides for a handover, it has to select the candidate AP from a considerable list of available nearby networks, inclusive of those that are either not located in the vehicles movement direction or for which the crossing time is not sufficient for handover activities (i.e., the cellular coverage area with respect to the vehicles speed of movement is trivial). From a list of potential candidate networks, selecting the most appropriate target candidate network for the handover activity is critical and is a must requirement for seamless mobility. Retaining the quality of service (QoS) of an ongoing session highly depends on the appropriate choice of the target candidate network for handover. the current work discusses a novel target network selection mechanism for potential handover activity in a heterogeneous network environment consisting LTE macro cells and Wi-Fi small cells, as per the proposal, concerned vehicles can self-select the target network and initiate the handover. Also, as the 
vehicle can choose the target network well in advance, it helps to improve the overall handover performance by reducing the handover delay.

Figure 1 illustrates a scenario where a vehicle is moving from point $\mathrm{A}$ to point $\mathrm{B}$ at the direction of the arrow. The macro LTE network overlays the multiple micro WiFi networks of limited coverage that are installed as road-side units (RSU). However, the micro networks provide higher bandwidth and are relatively cost effective than the macro network. The proposed technique seeks to avoid selecting a candidate network AP, which is either not lying on the direction of the vehicle or do not offer enough time to complete the handover when vehicle is crossing them.

In the scenario presented in Figure 1, we assume that the vehicle is currently under the coverage of the macro network at point $\mathrm{A}$. When it starts moving towards point $\mathrm{B}$, the macro BS communicates to it the information about the geographical coordinates (latitude and longitude) of the nearby microcells. The macro BS can gather this information through the Advanced Network Discovery and Selection Function (ANDSF) entity. The vehicle OBU sends an ANDSF query to ANDSF server (which may be accessed via macro cell eNB) inquiring the coordinates of available candidate APs in its vicinity. The ANDSF query also contains the vehicle location, speed and direction so that the ANDSF server response contains only APs available in the vicinity of the vehicle.

Let $N=A P_{1}, A P_{2}, A P_{3}, \ldots, A P_{m}$ be the set of all of the nearby micro-cell APs received from ANDSF server. For faster and efficient handover process, as per our proposal, out of all the APs in set $N$, the vehicle needs to select only the potential candidate (a) whose coverage area with respect to the speed of vehicle is considerable and (b) whose the distance to the vehicle current location is sufficient to finalize the selection before entering its coverage area. We also introduce the parameters, $\omega_{m}, \phi_{m}, \omega_{t h}$ and $\phi_{t h}$, considered for our proposal. $\omega_{m}$ and $\phi_{m}$ denote the geodistance between the target candidate AP physical location to the trajectory of movement and the remaining distance for the vehicle to reach its coverage (see Fig.1), while $\omega_{t h}$ and $\phi_{t h}$ represents threshold values of $\omega_{m}$ and $\phi_{m}$, respectively.

As it can be seen from the Fig.1, We estimate the distance between the AP and the road as:

$$
\omega_{m}=d_{i} \sin \alpha
$$

where $d_{i}$ is the geo-distance between the current geo-location of the vehicle and the geo-location of the target candidate $A P_{i}$. Given the spherical form of the earth, this geo-distance is estimated by using haversine formula [14], which is a function to calculate the great-cycle distance between any two points on Earth.

Let $\left(\operatorname{Lat}_{v}, \operatorname{Lon}_{v}\right)$ and $\left(\operatorname{Lat}_{A P i}, \operatorname{Lon}_{A P i}\right)$ denote the coordinates of vehicle and candidate AP respectively. We also denote latitude separation by $\Delta_{l a t}$ and longitude separation by $\Delta_{\text {lon }}$. The geo-distance, $d_{i}$ is calculated as follows [15]:

$$
\begin{array}{r}
\text { haversine }\left(\frac{d}{R}\right)=\text { haversine }\left(\Delta_{\text {lat }}\right)+\cos \left(\text { Lat }_{v}\right) * \\
\cos \left(\text { Lat }_{A P i}\right) * \text { haversine }\left(\Delta_{\text {lon }}\right)+\sin \alpha
\end{array}
$$

Where the angles are in radians and $R=6371 \mathrm{~km}$ [15], is the radius of the Earth. Furthermore, haversine function is given by:

$$
\text { haversine }(\delta)=\sin ^{2}\left(\frac{\delta}{2}\right)
$$

Thus (1) can be written as:

$$
\begin{aligned}
& \text { haversine }\left(\frac{d}{R}\right)=\sin ^{2}\left(\frac{\Delta_{l a t}}{2}\right)+\cos \left(l a t_{v}\right) * \\
& \cos \left(l_{\text {lat }}\right) * \sin ^{2}\left(\frac{\Delta_{l o n}}{2}\right)
\end{aligned}
$$

By replacing the right side of (3) by $h$, we get the value of $d$ as

$$
d_{i}=R * \text { haversine }{ }^{-1}\left(h_{i}\right)=2 R \sin \left(\sqrt{h_{i}}\right)
$$

In Equation 1, $\alpha$ is the angle comprised between the vector of movement of the vehicle and the straight line originating from $A P_{i}$ physical location, which could be derived from GPS information [9]. One can observe that, the lower the value $\omega_{m}$, the better the candidates network and the probability that an Access point $A P_{i}$ is selected for handover is formulated as follows:

$$
P\left(A P_{i}\right) \text { select }= \begin{cases}1, & \omega_{m}=0 \\ \frac{\omega_{m}}{R_{c}}, & 0<\omega_{m}<R_{c} \\ 0, & \omega_{m} \geq R_{c}\end{cases}
$$

Where $R_{c}$ denotes the coverage area radius.

In the current proposal, to accommodate vehicles moving at different speeds, we define $\omega_{t h}$ to indicate the minimum coverage distance for a vehicle traveling at a given speed category (slow, medium and high). In this context, please note that:

$$
\omega_{t h}<\omega_{m}<R_{c}
$$

The third parameter, $\phi_{m}$, gives us the estimated distance from the coverage area of the target candidate networks to the current location of the vehicle and can be calculated as:

$$
\phi_{m}=d_{i} \cos \alpha
$$

Obviously, higher the value of $\phi_{m}$ more the time the vehicle gets to finalize the selection of the candidate AP before it enters the coverage area of the target network. Once that is done, the vehicle can then immediately initiate the handover process with the target candidate AP on entering its coverage area. Successful early selection of the candidate AP (network) for handover can not only facilitate the betterment of the overall handover performance by reducing the handover latency but also reduce the chances of a failed handover. Lastly, the parameter $\phi_{t h}$ indicates the minimum threshold distance from the vehicles current location to the coverage area of the target network that is required to enable 
the vehicle to successfully complete the selection process. Equation 9 implies:

$$
\frac{\phi_{t h}}{v} \leq \tau
$$

where $\tau$ represents the time required for completing selection process.

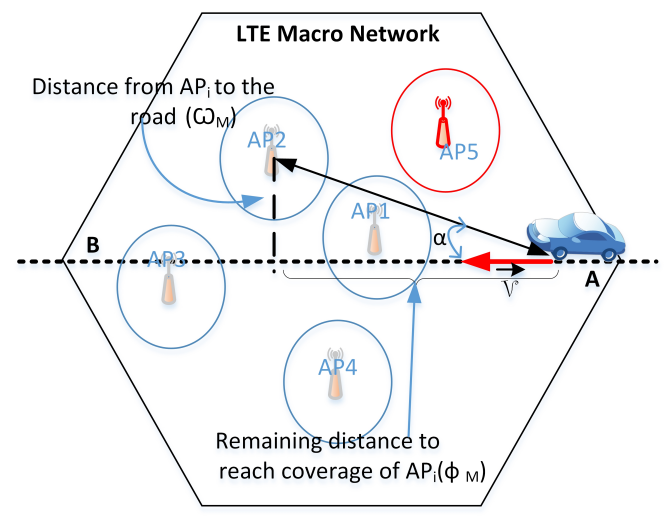

Fig. 1. Vehicle moving across multiple APs

\section{RESULTS AND DiscussiON}

\section{A. Fuzzy Logic}

We have used Fuzzy logic system to study and analyze our proposed network selection mechanism. Fuzzy logic has been used by researchers to effectively select the candidate network for handover [16-17]. In the current work, fuzzy logic is used to analyze how the candidate AP selection probability is effected by (a) the distance between the candidate AP and the vehicles movement trajectory and (b) the speed of movement of the vehicle. Note that the effect of the remaining distance to reach the coverage of the target $\mathrm{AP}$ is not considered in this implementation, it will be evaluated in our future work. In this work, the speed of movement values of the moving vehicle are approximated over regular intervals and are interpreted as low, medium and high. The Mamdani-type fuzzy logic controller is used here [18] to show the impact of both speed of movement and distance between a vehicles trajectory and AP on the selection of the candidate network.

In Figure 2 we introduce our proposed Fuzzy logic System (FLS) along with the considered input and output variables. The system, implemented in MatLab accepts two input variables, namely, distance between the target candidate $\mathrm{AP}$ and the trajectory of the vehicle movement and the speed of movement . Based on predetermined fuzzy if-then rules (Table I), the FLS work on these input values and the output value determines the probability of selecting a target candidate AP as per our proposed candidate network shortlisting/selection mechanism. The membership function of both input variables are presented in Figures 3 (for the distance) and 4 (for speed). Each input membership function has three linguistic variables: Low, Medium and High (Table II). Thus, the total number of fuzzy if-then rules is nine $\left(3^{2}\right)$ and are presented in Table I.

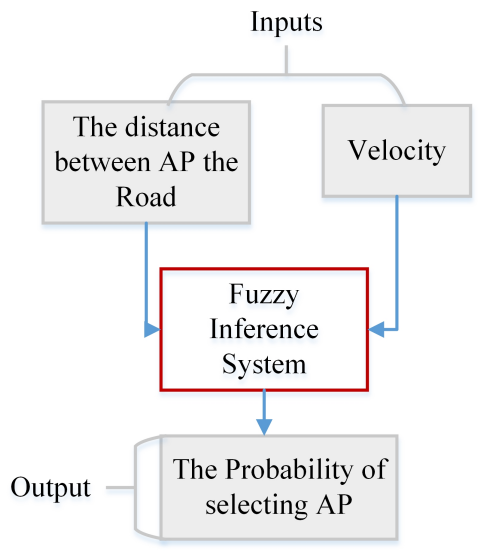

Fig. 2. The Fuzzy Inference System proposed as network selection model

For the simulation, we have considered small cells of $100 \mathrm{~m}$ radius and vehicular speed varying from 0 to $120 \mathrm{~km} / \mathrm{h}$. Table I provides the considered fuzzy if-then rules while Table II summarizes the input linguistic variables. For example, referring to the first cell of Table I, if the distance between target candidate AP and the vehicles movement trajectory is Low and the speed is also Low, then probability of selection of the target candidate AP is high. This implies that the target candidate AP is located near the future movement trajectory of the vehicle. Also, as vehicle is moving at low speed, it will likely spend reasonable time under the coverage area of the target candidate AP.

TABLE I

FUZZY RULES BASED NETWORK SELECTION

\begin{tabular}{|l|l|l|l|}
\hline Speed & Low & Medium & High \\
\hline Low & High & Medium & Low \\
Medium & Medium & Low & Low \\
High & Low & Low & Low \\
\hline
\end{tabular}

TABLE II

INPUT LINGUISTIC VARIABLES

\begin{tabular}{|l|l|l|l|}
\hline input variables & Low & Medium & High \\
\hline Distance $(m)$ & 030 & 3080 & 80100 \\
Speed & 030 & 3060 & 60120 \\
\hline
\end{tabular}

\section{B. Results}

The proposed FLS assessed the performance of our proposed network selection approach for heterogeneous networks. Through the MatLab simulations we analyzed how the probability of selecting the target candidate AP is affected by the vehicles speed variation as well as the distance from the target candidate AP (the coverage area) to the vehicles movement trajectory. Figure 5 shows the effect of the variation of distance between the target candidate AP and movement trajectory (the road) of the vehicle. As evident from the figure, for vehicular speeds between 30 and $60 \mathrm{Km} / \mathrm{h}$, less the distance between $\mathrm{AP}$ and the 


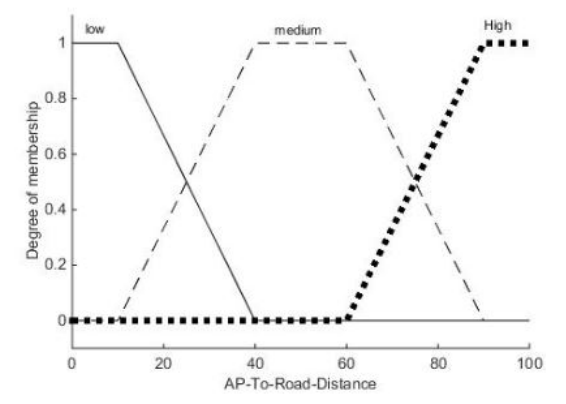

Fig. 3. Distance membership function

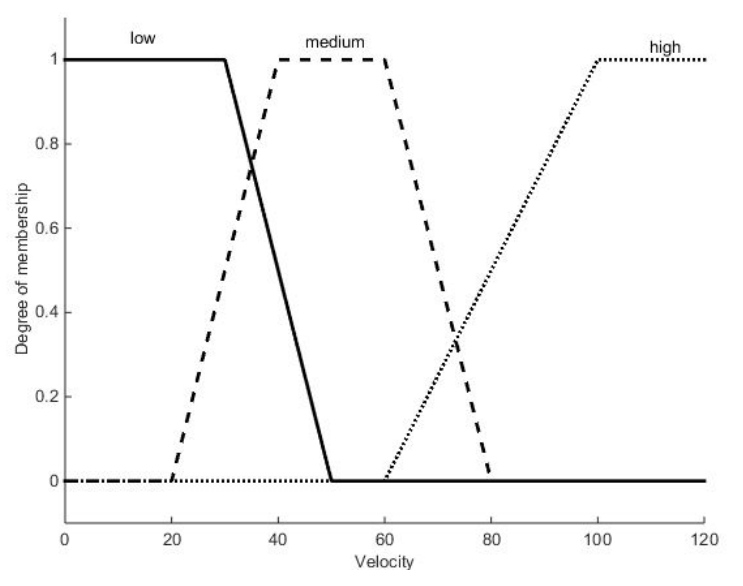

Fig. 4. Velocity membership function

vehicles movement trajectory, more/higher is the selection probability of a candidate network. On the contrary, the selection probability is always low for high speed vehicles. For instance, if the selection probability is 0.5 , for a vehicle moving at $30 \mathrm{~km} / \mathrm{h}$, a potential candidate AP should be located at less than $30 \mathrm{~m}$ from the road, while this distance is limited to $15 \mathrm{~m}$ when the vehicle speed is $60 \mathrm{~km} / \mathrm{h}$. Figure 6 demonstrates the combined influence of distance and speed to the target candidate network selection probability. As can be seen in the figure, the maximum selection probability (indicated by the top red colored surface area) is obtained when both the vehicles speed and the distance between the AP coverage area and movement trajectory are the lowest. On the contrary, the lowest selection probability (indicated by the orange colored surface area at the bottom) is associated with the highest values for both the input variables. This shows clearly how the proposed approach provides a simple and reliable network selection, which may reduce the overall handover latency since the network selection activities start in advance, and where the hand-off decision could be based on a simple trade-off between speed and gaining small cells benefits.

\section{CONCluding Remarks}

A novel target network selection mechanism for handover in a heterogeneous network environment consisting of LTE

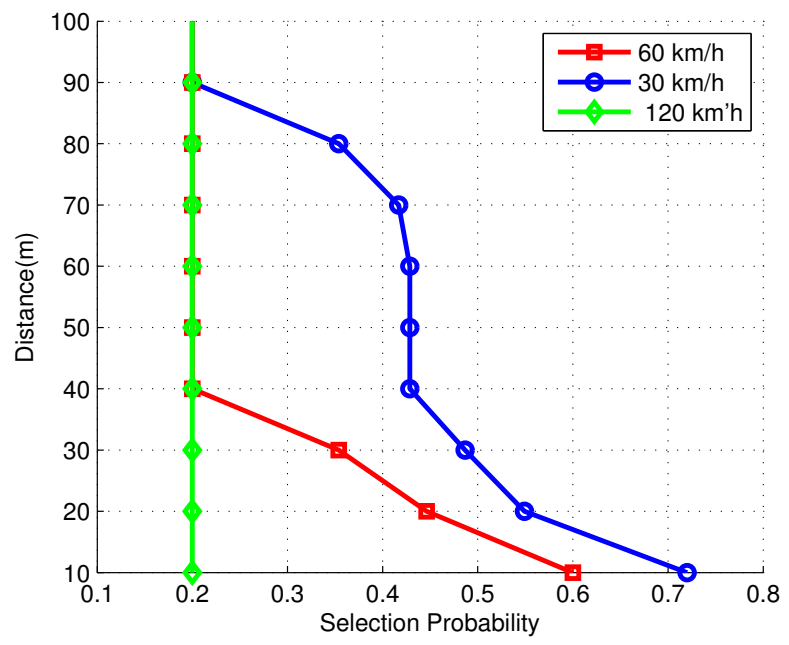

Fig. 5. Effect of the distance variation for a various fixed values of the velocity

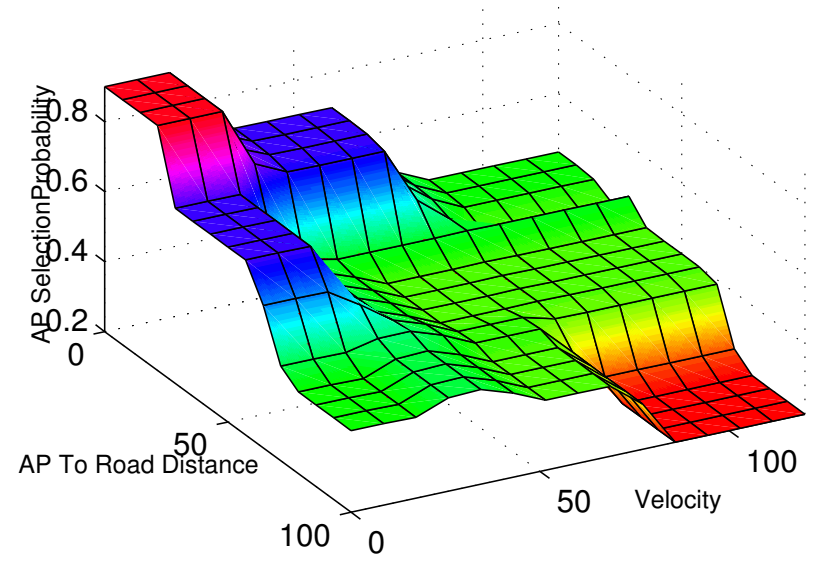

Fig. 6. Illustrating the effect of mobility and the distance between AP to the vehicle movement trajectory

macro and WiFi small cells is proposed in this paper. The primary objective is to reduce the overall handover delay by performing network selection in advance, and to avoid unsuccessful handovers resulting in Ping-Pong effects. In the proposal, concerned vehicles can self-select the target network, which lies in its direction of movement and for which the dwelling time of the vehicle within its coverage area is considerable. A fuzzy logic inference system is used to decide whether a particular candidate network is suitable for handover. Immediate Future work on this topic will explore the network selection approach in a multi-tier heterogeneous environment consisting of macro, pico, femto and other smalls cells.

\section{REFERENCES}

[1] Y.-H. Cheng, et al., "A Fast Safety Message Transmission Mechanism for Heterogeneous Vehicular Networks," Appl. Math, NSP, vol. 9, no. 1L, pp. 219-228, 2015. 
[2] R. Gartner Inc., Predicts 2015: The Internet of Things[nline] available: http://www.gartner.com/ document/2952822. [Accessed: Feb. 2016]

[3] G. B. Seraphin and S. Ramesh, "QoS Provisioning in Vertical Handover of WiMAX/WLAN Overlay Network for Fixed and Mobile Users," Wireless Communication, CiiT, vol. 5, pp. 158-162, 2013.

[4] V. Friderikos, et al., "Challenges of 5G Networking in Access and Core Networks," Handbook of Research on Redesigning the Future of Internet Architectures, p. 161, 2015.

[5] T. T. Jianlin Guo, Jinyun Zhang, "Location Aware Fast Handover Between WiMax andWiFi Networks,"MITSUBISHI ELECTRIC RESEARCH LABORATORIES, http://www. merl. com, 2010.

[6] ETSITS124312, "Universal Mobile Telecommunications System(UMTS), LTE; Access Network Discovery and Selection Functions (ANDSF) Management Object (MO) 3GPP TS 24.312 version 12.10.0 Release 12, October 2015.

[7] Sayan K. Ray, H. Sirisena, D. Deka, "Fast and Reliable Target Base Station Selection Scheme for Mobile WiMAX Handover", in Proc. of 9th Australasian Telecommunication Networks and Applications Conference (ATNAC 2012), Brisbane, Australia, 7-9 Nov 2012, pp. 1-6, DOI: 10.1109/ATNAC.2012.6398054

[8] Sayan K. Ray, H. Sirisena, D. Deka, "LTE-Advanced Handover: An Orientation Matching-based Fast and Reliable Approach", in Proc of 38th IEEE Conference on Local Computer Networks (LCN 2013), Sydney, Australia,21-24 Oct 2013, pp. 280-283, DOI: 10.1109/LCN.2013.6761249

[9] Xi, L., Z. Heli, and X. Quansheng, "Optimal access scheme for mobile vehicular small cells in layered heterogeneous networks", in Proc. of 4th IEEE International Conference on Network Infrastructure and Digital Content (IC-NIDC),Beijing, China, 19-21 Sept. 2014,pp. 4650, DOI:10.1109/ICNIDC.2014.7000263.

[10] Y. Xu, L. Li, B.-H. Soong, and C. Li, "Fuzzy Q-learning based vertical handoff control for vehicular heterogeneous wireless network," in Proc.of IEEE International Conference on Communications (ICC), Sydney, Australia, 2014, pp. 5653-5658, DOI:10.1109/ICC.2014.6884222.

[11] A. Mehbodniya, F. Kaleem, K. K. Yen, and F. Adachi, ”A fuzzy MADM ranking approach for vertical mobility in next generation hybrid networks," in Ultra Modern Telecommunications and Control Systems and Workshops (ICUMT), 2012 4th International Congress on,St. Petersburg, Russia, 3-5 Oct. 2012, pp. 262-267, DOI: 10.1109/ICUMT.2012.6459676.

[12] L. A. Zadeh, "Fuzzy sets as a basis for a theory of possibility." Fuzzy sets and systems, Elsevier, vol. 1, no. 1, pp. 328, 1978.

[13] B. Van Quang, R. V. Prasad, and I. Niemegeers, "A survey on handoffslessons for $60 \mathrm{GHz}$ based wireless systems," Communications Surveys \& Tutorials, IEEE, vol. 14, no.1, pp. 64-86, 2012.

[14] Mwemezi, J.J. and Y. Huang, "Optimal facility location on spherical surfaces: algorithm and application", New York Science Journal, Vol. 2, no. 7, 2011.

[15] J. Montavont and T. Noel, "IEEE 802.11 handovers assisted by GPS information," in Proc. of IEEE International Conference on Wireless and Mobile Computing, Networking and Communications,Montreal, Canada, 19-21 June 2006, pp. 166-172, DOI: $10.1109 /$ WIMOB.2006.1696358

[16] N. Abbas and J. J. Saade, "A fuzzy logic based approach for network selection in WLAN/3G heterogeneous network," in Proc. of 12th IEEE Consumer Communications and Networking Conference (CCNC), Las Vegas, NV, USA, 9-12 Jan. 2015, pp. 631-636, DOI:10.1109/CCNC.2015.7158053.

[17] J. Kim, et al., "Fuzzy logic based handoff scheme for heterogeneous vehicular mobile networks," in Proc.of IEEE International Conference on High Performance Computing and Simulation (HPCS), Bologna, Italy, 21-25 July 2014, pp. 863-870, DOI:10.1109/HPCSim.2014.6903780.

[18] O. Cordn, "A historical review of evolutionary learning methods for Mamdani-type fuzzy rule-based systems: Designing interpretable genetic fuzzy systems," International Journal of Approximate Reasoning, Elsevier, vol. 52, no. 6, pp. 894-913, September 2011. 